B. V. Normenyo, S. E. Rihane, A. Togbe

\title{
FERMAT AND MERSENNE NUMBERS IN THE $k$-PELL SEQUENCE
}

\author{
B. V. Normenyo, S. E. Rihane, A. Togbe. Fermat and Mersenne numbers in the $k$-Pell sequence, \\ Mat. Stud. 56 (2021), 115-123.
}

For an integer $k \geq 2$, let $\left(P_{n}^{(k)}\right)_{n \geq 2-k}$ be the $k$-generalized Pell sequence, which starts with $0, \ldots, 0,1$ ( $k$ terms) and each term afterwards is defined by the recurrence $P_{n}^{(k)}=2 P_{n-1}^{(k)}+$ $P_{n-2}^{(k)}+\cdots+P_{n-k}^{(k)}$, for all $n \geq 2$. For any positive integer $n$, a number of the form $2^{n}+1$ is referred to as a Fermat number, while a number of the form $2^{n}-1$ is referred to as a Mersenne number. The goal of this paper is to determine Fermat and Mersenne numbers which are members of the $k$-generalized Pell sequence. More precisely, we solve the Diophantine equation $P_{n}^{(k)}=2^{a} \pm 1$ in positive integers $n, k, a$ with $k \geq 2, a \geq 1$. We prove a theorem which asserts that, if the Diophantine equation $P_{n}^{(k)}=2^{a} \pm 1$ has a solution $(n, a, k)$ in positive integers $n, k, a$ with $k \geq 2, a \geq 1$, then we must have that $(n, a, k) \in\{(1,1, k),(3,2, k),(5,5,3)\}$. As a result of our theorem, we deduce that the number 1 is the only Mersenne number and the number 5 is the only Fermat number in the $k$-Pell sequence.

1. Introduction. Let $k \geq 2$ be an integer. We consider a generalization of Pell sequence called the $k$-generalized Pell sequence $P_{n}^{(k)}$, or, for simplicity, the $k$-Pell sequence, defined as

$$
P_{n}^{(k)}=2 P_{n-1}^{(k)}+P_{n-2}^{(k)}+\cdots+P_{n-k}^{(k)}, \quad n \geq 2,
$$

with initial conditions $P_{-(k-2)}^{(k)}=P_{-(k-3)}^{(k)}=\cdots=P_{-1}^{(k)}=P_{0}^{(k)}=0$, and $P_{1}^{(k)}=1$. This generalization is a family of sequences in the sense that each value of $k$ engenders a distinct sequence. For instance, when we set $k=2$, we arrive at the usual Pell sequence. There is an analogous definition for $k$-generalized Fibonacci sequence $F_{n}^{(k)}$.

In recent times, a number of papers have been written aimed at determining members of certain sequences which are powers of 2 . Bravo and Luca solved the Diophantine equations $L_{n}+L_{m}=2^{a}$ and $F_{n}+F_{m}=2^{a}$ in [5] and [4], respectively. The idea was extended to generalizations of the Lucas sequences. This can be seen, for example, in [6] and [7].

A Fermat number is a number of the form $2^{n}+1$. Its first few terms are $3,5,9,17$, $33, \ldots$. A Mersenne number is a number of the form $2^{n}-1$, where $n$ is a positive integer. The Mersenne numbers consist of all $1 \mathrm{~s}$ in base 2 , and are therefore binary repunits. The first few Mersenne numbers are 1, 3, 7, 15, 31, 63, 127, 255, .... Some properties of these numbers have been studied. One can cite [11], [12].

In this paper, we seek to find Fermat and Mersenne numbers which are members of the $k$-Pell sequence. More precisely, we study the Diophantine equation

$$
P_{n}^{(k)}=2^{a} \pm 1,
$$

2010 Mathematics Subject Classification:11B39, 11J86.

Keywords: $k$-Pell number; Fermat number; Mersenne number; Fibonacci number; linear form in logarithms; reduction algorithm.

doi:10.30970/ms.56.2.115-123

(C) B. V. Normenyo, S. E. Rihane, A. Togbe, 2021 
where $n, k$ and $a$ are positive integers with $k \geq 2$ and $a \geq 1$. We will establish the following results.

Theorem 1. All the solutions of the Diophantine equation

$$
P_{n}^{(k)}=2^{a} \pm 1
$$

in positive integers $n, k, a$ with $k \geq 2$ and $a \geq 1$ are

$$
(n, a, k) \in\{(1,1, k),(3,2, k),(5,5,3)\} .
$$

The next results are straightforward consequences of Theorem 1 .

Corollary 1. The number 1 is the only Mersenne number in the k-Pell sequence.

Corollary 2. The number 5 is the only Fermat number in the $k$-Pell sequence.

This paper is organized as follows. In the next section, we develop the tools that will be used to prove Theorem 1. This proof will be done in last section in four steps. In the first step, we will setup the problem, find the parametric solutions, and bound $n$ in terms of $a$. The second step consists in finding an upper bound of $n$ in term of $k$. For the third step, we consider lower values of $k$, i.e. $2 \leq k \leq 320$, and solve equation (1) in this range by finding the non-parametric solution. In the final step, we take $k \geq 321$ and prove that we have no other solution.

2. The tools. This section is devoted to collecting definitions, notations, properties and results which will be used throughout this paper.

2.1. Linear forms in logarithms. For any nonzero algebraic number $\eta$ of degree $d$ over $\mathbb{Q}$, whose minimal polynomial over $\mathbb{Z}$ is $a \prod_{j=1}^{d}\left(X-\eta^{(j)}\right)$, we denote by

$$
h(\eta)=\frac{1}{d}\left(\log |a|+\sum_{j=1}^{d} \log \max \left\{1,\left|\eta^{(j)}\right|\right\}\right)
$$

the usual absolute logarithmic height of $\eta$. In particular, if $\eta=p / q$ is a rational number with $\operatorname{gcd}(p, q)=1$ and $q>0$, then $h(\eta)=\log \max \{|p|, q\}$. The following properties of the logarithmic height function $h(\cdot)$, which will be used in the next sections without special reference, are well-known:

$$
\begin{aligned}
h(\eta \pm \gamma) & \leq h(\eta)+h(\gamma)+\log 2 \\
h\left(\eta \gamma^{ \pm 1}\right) & \leq h(\eta)+h(\gamma), \\
h\left(\eta^{s}\right) & =|s| h(\eta) \quad(s \in \mathbb{Z}) .
\end{aligned}
$$

The first tool we need is the following result due to Matveev [17]. Here, we use the version due to Bugeaud, Mignotte, and Siksek [9, Theorem 9.4].

Theorem 2. Let $\eta_{1}, \ldots, \eta_{s}$ be real algebraic numbers and let $b_{1}, \ldots, b_{s}$ be nonzero integers. Let $d_{\mathbb{K}}$ be the degree of the number field $\mathbb{K}:=\mathbb{Q}\left(\eta_{1}, \ldots, \eta_{s}\right)$ over $\mathbb{Q}$ and let $A_{j}$ be a positive real number satisfying

$$
A_{j}=\max \left\{d_{\mathbb{K}} h(\eta),|\log \eta|, 0.16\right\} \quad \text { for } \quad j=1, \ldots, s .
$$

Assume that $B \geq \max \left\{\left|b_{1}\right|, \ldots,\left|b_{s}\right|\right\}$. If $\eta_{1}^{b_{1}} \cdots \eta_{s}^{b_{s}}-1 \neq 0$, then

$$
\left|\eta_{1}^{b_{1}} \cdots \eta_{s}^{b_{s}}-1\right| \geq \exp \left(-1.4 \cdot 30^{s+3} \cdot s^{4.5} \cdot d_{\mathbb{K}}^{2}\left(1+\log d_{\mathbb{K}}\right)(1+\log B) A_{1} \cdots A_{s}\right) .
$$


2.2. The reduction algorithm. Our second tool is a version of the reduction method of Baker and Davenport. We use a slight variant of the version given by Dujella and Pethö [10].

Lemma 1. Let $M$ be a positive integer, $p / q$ a convergent of the continued fraction of the irrational $\gamma$ such that $q>6 M$, and $A, C, \mu$ some real numbers with $A>0$ and $B>1$. Let

$$
\varepsilon=\|\mu q\|-M \cdot\|\gamma q\|,
$$

where $\|\cdot\|$ denotes the distance from the nearest integer. If $\varepsilon>0$, then there is no solution of the inequality

$$
0<u \gamma-v+\mu<A B^{-w}
$$

in positive integers $u, v$ and $w$ with

$$
u \leq M \quad \text { and } \quad w \geq \frac{\log (A q / \varepsilon)}{\log C} .
$$

We see that Lemma 1 cannot be applied when $\mu=0$ (since then $\varepsilon<0$ ). For this case, we use the following well-known technical result from Diophantine approximation, known as Legendre's criterion which is our third tool. This comes from the theory of continued fractions (see [13], pages 30 and 37).

Lemma 2. Let $p_{i} / q_{i}$ be the convergents of the continued fraction $\left[a_{0}, a_{1}, \ldots\right]$ of the irrational number $\gamma$. Let $M$ be a positive integer and put $a_{M}:=\max \left\{a_{0}, a_{1}, \ldots, a_{N+1}\right\}$, where $N \in \mathbb{N}$ is such that $q_{N} \leq M<q_{N+1}$. If $x, y \in \mathbb{Z}$ with $x>0$, then

$$
|x \gamma-y|>\frac{1}{\left(a_{M}+2\right) x}, \quad \text { for all } x<M
$$

2.3. Useful Lemmas. We conclude this section by recalling two lemmas that we need in this work:

Lemma 3 ([18], Lemma 7). If $m \geq 1, T>\left(4 m^{2}\right)^{m}$ and $T>y /(\log y)^{m}$, then

$$
y<2^{m} T(\log T)^{m} .
$$

Lemma 4 ([19], Lemma 2.2). Let $d, x \in \mathbb{R}$ and $0<d<1$. If $|x|<d$, then

$$
|\log (1+x)|<\frac{-\log (1-d)}{d}|x| .
$$

2.4. On $k$-Pell sequence. In this subsection, we recall some facts and properties of the $k$-Pell sequences which will be used later. The characteristic polynomial of these sequences is

$$
\Psi_{k}(x)=x^{k}-2 x^{k-1}-x^{k-2}-\cdots-x-1 .
$$

In [3], Bravo et al. show that $\Psi_{k}(x)$ is irreducible over $\mathbb{Q}[x]$ and has just one zero $\alpha(k)$ outside the unit circle. It is real and positive so it satisfies $\alpha(k)>1$. The other zeros are strictly inside the unit circle. Furthermore, in the same paper, they show that

$$
\varphi^{2}\left(1-\varphi^{-k}\right)<\alpha(k)<\varphi^{2}, \quad \text { for all } k \geq 2,
$$


where $\varphi=(1+\sqrt{5}) / 2$. To simplify the notation, we omit the dependence on $k$ of $\alpha$. For $k \geq 2$, let

$$
g_{k}(x):=\frac{x-1}{(k+1) x^{2}-3 k x+k-1}=\frac{x-1}{k\left(x^{2}-3 x+1\right)+x^{2}-1} .
$$

In [2], Bravo and Hererra proved that

$$
0.276<g_{k}(\alpha)<0.5 \text { and }\left|g_{k}\left(\alpha^{(i)}\right)\right|<1,2 \leq i \leq k
$$

hold, where $\alpha:=\alpha^{(1)}, \alpha^{(2)}, \ldots, \alpha^{(k)}$ are all the zeros of $\Psi_{k}(x)$. So, the number $g_{k}(\alpha)$ is not an algebraic integer. In addition, they proved that the logarithmic height of $g_{k}$ satisfies

$$
h\left(g_{k}(\alpha)\right)<4 k \log \varphi+k \log (k+1) \quad \text { for all } k \geq 2 .
$$

With the above notation, Bravo and Hererra showed in [3] that

$$
P_{n}^{(k)}=\sum_{i=1}^{k} g_{k}\left(\alpha^{(i)}\right) \alpha^{(i)^{n-1}} \quad \text { and } \quad\left|P_{n}^{(k)}-g_{k}(\alpha) \alpha^{n-1}\right|<\frac{1}{2}
$$

hold, for all $n \geq 1$ and $k \geq 2$. Furthermore, for $n \geq 1$ and $k \geq 2$, it was showed in [3] that

$$
\alpha^{n-2} \leq P_{n}^{(k)} \leq \alpha^{n-1}
$$

3. The proof of Theorem 1. In this section, we will prove Theorem 1 in four steps. Let us start with the first step.

3.1. Setup. If $a=1$, then (1) imply that $P_{n}^{(k)}=1$ or $P_{n}^{(k)}=3$. Hence $(n, a, k)=(1,1, k)$ with $k \geq 2$ is the only solutions of the Diophantine equation (1) in this case.

Note that for $2 \leq n \leq k+1$, we have $P_{n}^{(k)}=F_{2 n-1}$, where $F_{m}$ is the $m$-th Fibonacci number (see [14]), so the equation (1) becomes

$$
F_{2 n-1} \mp 1=2^{a} .
$$

In [8], Bugeaud et al. showed that the only nonnegative integer solutions $(n, y, m)$ of equations $F_{n} \pm 1=y^{m}$ with $m \geq 2$ are

$$
\begin{aligned}
& F_{4}+1=3+1=2^{2}, \\
& F_{6}+1=8+1=3^{2}, \\
& F_{5}-1=5-1=2^{2} .
\end{aligned}
$$

As a consequence of the above, $(n, a)=(3,2)$ is the only nonnegative integer solution of equation (10). So, $(n, a, k)=(3,2, k)$ with $k \geq 2$ is a solution of the Diophantine equation (1).

In the remainder of this paper, we suppose that $n \geq k+2, n \geq 4$ and $a \geq 2$. Using equation (1), we obtain the inequality

$$
2^{a-1} \leq 2^{a}-1 \leq 2^{a} \pm 1=P_{n}^{(k)}<\alpha^{n-1}<\varphi^{2(n-1)},
$$

from which we infer $0.7 a+0.2<n$. Furthermore, we get

$$
\varphi^{n-2}<\alpha^{n-2}<P_{n}^{(k)}=2^{a} \pm 1 \leq 2^{a}+1<2^{a+1},
$$

from which we infer $n<1.5 a+2.8$. Consequently, we find that

$$
0.7 a+0.2<n<1.5 a+2.8 .
$$


3.2. An inequality for $n$ versus $k$. In this subsection, we shall be preoccupied with determining an upper bound for $n$ in terms of $k$. To begin with, observe that equation (1), when expressed in the form

$$
2^{a}=P_{n}^{(k)} \mp 1
$$

produces

$$
\left|2^{a}-g_{k}(\alpha) \alpha^{n-1}\right| \leq \frac{3}{2},
$$

which, upon dividing through by $g_{k}(\alpha) \alpha^{n-1}$, yields

$$
\left|2^{a} \cdot \alpha^{-(n-1)} \cdot\left(g_{k}(\alpha)\right)^{-1}-1\right|<\frac{3}{2 \cdot 0.276 \cdot \alpha^{n-1}}<\frac{14.3}{\alpha^{n}}
$$

where we have used the fact that $\alpha<\varphi^{2}$ and $g_{k}(\alpha)>0.276$. Let

$$
\Gamma_{1}=2^{a} \cdot \alpha^{-(n-1)} \cdot\left(g_{k}(\alpha)\right)^{-1}-1 .
$$

We claim that $\Gamma_{1} \neq 0$. To prove our claim, we suppose that $\Gamma_{1}=0$. This means that

$$
g_{k}(\alpha)=2^{a} \cdot \alpha^{-(n-1)},
$$

which implies that $g_{k}(\alpha)$ is an algebraic integer, which is a contradiction. Hence, $\Gamma_{1} \neq 0$. We apply Theorem 2 to $\Gamma_{1}$ given by (15). To do this, we take

$$
\begin{gathered}
\eta_{1}:=2, \quad \eta_{2}:=\alpha, \quad \eta_{3}:=g_{k}(\alpha), \\
b_{1}:=a, \quad b_{2}:=-(n-1), \quad \text { and } \quad b_{3}:=-1 .
\end{gathered}
$$

The algebraic numbers $\eta_{1}, \eta_{2}, \eta_{3}$ are elements of the field $\mathbb{K}:=\mathbb{Q}(\alpha)$ with $d_{\mathbb{K}}=k$. We find that

$$
h\left(\eta_{1}\right)=\log 2, \quad h\left(\eta_{2}\right)=\frac{\log \alpha}{k},
$$

and

$$
h\left(\eta_{3}\right) \leq 4 k \log \varphi+k \log (k+1)<4.5 k \log k,
$$

for all $k \geq 2$. Thus, we take

$$
A_{1}:=k \log 2, \quad A_{2}:=2 \log \varphi, \quad \text { and } \quad A_{3}:=4.5 k^{2} \log k .
$$

In addition, we take $B:=1.5 n$, using inequality (11). Furthermore, we observe that the inequalities $1+\log k<2.5 \log k$ and $1+\log (1.5 n)<2.1 \log n$ hold, for all $k \geq 2$ and $n \geq 4$, respectively. Applying Theorem 2 to $\Gamma_{1}$ given by (15) and using inequality (14), we obtain

$$
n<2.36 \cdot 10^{12} \cdot k^{5} \log ^{2} k \cdot \log n .
$$

In the notation of Lemma 3, inequality (16) implies that $m=1, T=2.36 \cdot 10^{12} \cdot k^{5} \log ^{2} k$, and $y=n$. Thus, we get

$$
\begin{gathered}
n<2 \cdot 2.36 \cdot 10^{12} \cdot k^{5} \log ^{2} k \log \left(2.36 \cdot 10^{12} \cdot k^{5} \log ^{2} k\right)< \\
<\left(4.72 \cdot 10^{12} \cdot k^{5} \log ^{2} k\right)(46 \log k)<2.2 \cdot 10^{14} k^{5} \log ^{3} k .
\end{gathered}
$$

The result established in this subsection is summarized in the following lemma. 
Lemma 5. If $(n, a, k)$ is a solution in integers of equation (1) with $k \geq 2, n \geq k+2$ and $a \geq 2$, then the inequality

$$
n<2.2 \cdot 10^{14} k^{5} \log ^{3} k
$$

holds.

3.3. The case $2 \leq k \leq 320$. In this subsection, we study the problem when $k \in[2,320]$. Our main tool is Lemma 1 . We begin by putting

$$
\Lambda_{1}:=\log \left(\Gamma_{1}+1\right)=a \log 2-(n-1) \log \alpha-\log \left(g_{k}(\alpha)\right)
$$

It is easy to see that $\left|\Gamma_{1}\right|<0.12$ for $n \geq 10$. As a result, using inequality (14), we obtain

$$
\left|\Lambda_{1}\right|=\left|\log \left(\Gamma_{1}+1\right)\right|<\frac{-\log (1-0.12)}{0.12}\left|\Gamma_{1}\right|<15.3 \cdot \alpha^{-n} .
$$

Therefore, using $\alpha>\varphi$, we get

$$
0<\left|a \cdot \frac{\log 2}{\log \alpha}-n+\left(1-\frac{\log g_{k}(\alpha)}{\log \alpha}\right)\right|<31.8 \cdot \alpha^{-n} .
$$

In order to apply Lemma 1 to the above inequality, we take

$$
u:=a, \gamma:=\frac{\log 2}{\log \alpha}, v:=n, \mu:=1-\frac{\log g_{k}(\alpha)}{\log \alpha}, A:=31.8, B:=\alpha, \omega:=n .
$$

We have $\gamma \notin \mathbb{Q}$ since if we assume the contrary, then there exist coprime integers $a_{1}$ and $a_{2}$ such that $\gamma=a_{1} / a_{2}$, which means that $\alpha^{a_{1}}=2^{a_{2}}$. Let $\sigma \in \operatorname{Gal}(\mathbb{K} / \mathbb{Q})$, the Galois group of the extension $\mathbb{K} / \mathbb{Q}$, such that $\sigma(\alpha)=\alpha_{i}$, for some $i \in\{2, \ldots, k\}$. Applying this to the above relation and taking absolute values we get $1<2^{a_{2}}=\left|\alpha_{i}\right|<1$, which is a contradiction. Moreover, we note that $a \leq 1.5 n \leq\left\lfloor 3.3 \cdot 10^{14} k^{5} \log ^{3} k\right\rfloor$. Thus, for each $k \in[2,320]$, we take $M_{k}=\left\lfloor 3.3 \cdot 10^{14} k^{5} \log ^{3} k\right\rfloor$ and we find a good approximation of $\gamma$ and a convergent $p_{\ell} / q_{\ell}$ of the continued fraction of $\gamma$ such that $q_{\ell}>6 M_{k}$ and $\varepsilon=\varepsilon(k)=\|\mu q\|-M_{k}\|\gamma q\|>0$. Appealing to a computer program in Mathematica, Lemma 1 applied to $\Lambda_{1}$ gives us $n \leq$ 88.5346 .

Hence, we deduce that the possible solutions $(n, a, k)$ of equation (1) for which $k \in[2,320]$ satisfy $k+2 \leq n \leq 88$ and $1 \leq a \leq 132$. Finally, using a brute force search with Mathematica in this ranges we get the only solution $P_{5}^{(3)}=2^{5}-1$.

3.4. Large values of $k$. In this subsection, we will consider $k \geq 321$. The work will be done in two steps.

3.4.1. An absolute upper bound on $k$. Here, we will show the following lemma that consists in bounding $k$ and $n$.

Lemma 6. If $(n, a, k)$ is a solution of the Diophantine equation (1) with $k>320$ and $n \geq k+2$, then $k$ and $n$ are bounded as

$$
k<4.8 \cdot 10^{15} \text { and } n<2.7 \cdot 10^{97} \text {. }
$$


Proof. For $k>320$, it is easy to check that

$$
n<2.2 \cdot 10^{14} k^{5} \log ^{3} k<\varphi^{k / 2} .
$$

Thus, from [2], $g_{n}(\alpha) \alpha^{n}$ can be rewritten as

$$
g_{n}(\alpha) \alpha^{n}=\frac{\varphi^{2 n}}{\varphi+2}(1+\zeta), \quad \text { where }|\zeta|<\frac{4}{\varphi^{k / 2}} .
$$

Combining (21) and (13), the inequality

$$
\begin{aligned}
&\left|2^{a}-\frac{\varphi^{2 n}}{\varphi+2}\right| \leq \mid 2^{a}-g_{k}(\alpha) \alpha^{n}\left|+\frac{\varphi^{2 n}}{\varphi+2}\right| \zeta \mid \leq \frac{\varphi^{2 n}}{\varphi+2}\left(\frac{3(\varphi+2)}{2 \varphi^{2 n}}+\frac{4}{\varphi^{k / 2}}\right) \leq \\
& \leq \frac{\varphi^{2 n}}{\varphi+2}\left(\frac{3 \varphi+10}{\varphi^{k / 2}}\right) \leq \frac{15 \varphi^{2 n}}{(\varphi+2) \varphi^{k / 2}}
\end{aligned}
$$

is obtained. Note that in the above inequalities, we have used the fact that $n \geq k+2$ implies $2 n>k / 2$, and so $2 \varphi^{2 n}>\varphi^{k / 2}$. Hence, from the above inequality, we obtain

$$
\left|(\varphi+2) \cdot 2^{a} \cdot \varphi^{-2 n}-1\right|<\frac{15}{\varphi^{k / 2}} .
$$

We will apply Theorem 2 to obtain a lower bound to the left-hand side of inequality (22). We take

$$
t:=3, \quad\left(\eta_{1}, b_{1}\right):=(\varphi+2,1), \quad\left(\eta_{2}, b_{2}\right):=(2, a), \quad\left(\eta_{3}, b_{3}\right):=(\varphi,-2 n),
$$

and

$$
\Gamma_{2}:=(\varphi+2) \cdot 2^{a} \cdot \varphi^{-2 n}-1 .
$$

Suppose that $\Gamma_{2}=0$, then

$$
2^{a}(\varphi+2)=\varphi^{2 n} .
$$

Conjugating the above relation in $\mathbb{Q}(\sqrt{5})$, we find that

$$
1<2^{a}(\bar{\varphi}+2)=\bar{\varphi}^{2 n}<1,
$$

where $\bar{\varphi}=(1-\sqrt{5}) / 2$. Therefore $\Gamma_{2} \neq 0$.

Since $\eta_{1}, \eta_{2}, \eta_{3} \in \mathbb{K}:=\mathbb{Q}(\sqrt{5})$, we see that $d_{\mathbb{K}}=2$. As $a \leq 1.5 n$, we take $B:=2 n=$ $\max \{a, 2 n, 1\}$. On the other hand, the fact that

$$
h\left(\eta_{1}\right) \leq h(\varphi)+h(2)+\log 2 \leq \frac{\log \varphi}{2}+2 \log 2<1.7,
$$

and

$$
h\left(\eta_{2}\right)=\log 2, \quad h\left(\eta_{3}\right)=\frac{\log \varphi}{2}
$$

imply that we can take

$$
A_{1}:=3.4, \quad A_{2}:=2 \log 2 \quad \text { and } \quad A_{3}:=\log \varphi .
$$

So, Theorem 2 tells us that

$$
\left|\Gamma_{2}\right|>\exp \left(-5.06 \cdot 10^{12} \log n\right),
$$


where we have used the fact that $1+\log 2 n<2.3 \log n$, for all $n \geq 4$. Putting (22) and (23) together, it follows that

$$
k<2.11 \cdot 10^{13} \log n<2.11 \cdot 10^{13} \log \left(2.2 \cdot 10^{14} k^{5} \log ^{3} k\right),
$$

where we have used Lemma 5. The desired result comes from solving the above inequality and using again Lemma 5.

3.4.2. Reducing the bound on $k$. Now, we try to reduce the obtained bounds. Put

$$
\Lambda_{2}:=a \log 2-2 n \log \varphi-\log (\varphi+2)=\log \left(\Gamma_{2}+1\right) .
$$

Since $k>320$, then from (22) we get $\left|\Gamma_{2}\right|<0.01$. Hence, by Lemma 4 , we conclude that

$$
\left|\Lambda_{2}\right|<-\frac{\log 0.99}{0.01} \cdot\left|\Gamma_{2}\right|<15.1 \cdot \varphi^{-k / 2}
$$

and so

$$
\left|a \cdot \frac{\log 2}{\log \varphi}-2 n-\frac{\log (\varphi+2)}{\log \varphi}\right|<31.4 \cdot \varphi^{-k / 2} .
$$

We apply Lemma 1 with the parameters

$$
\gamma:=\frac{\log 2}{\log \varphi}, \quad \mu:=\frac{\log (\varphi+2)}{\log \varphi}, \quad \text { and } \quad(A, B):=(31.4, \varphi) .
$$

Furthermore, since $a<1.5 n$ and $n<2.7 \cdot 10^{97}$ by Lemma 6 , we can take $M:=4.05 \cdot 10^{97}$. Let

$$
\left[a_{0}, a_{1}, a_{2}, a_{3}, \ldots\right]=[1,2,3,1,2,3,2,4,2,1,2,11,2,1,11,1,1,134, \ldots]
$$

be the continued fraction of $\gamma$. With the help of Mathematica, we find that $q_{194}$ satisfies the conditions of Lemma 1. Furthermore, according to Lemma 1 we get $k<984$. With this new upper bound on $k$, we get

$$
n<6.7 \cdot 10^{31} .
$$

We now repeat the process above but with $M=1.1 \cdot 10^{32}$, we obtain that $q_{71}$ satisfies the conditions of Lemma 1 and so $k \leq 344$. Hence, we deduce that

$$
n<2.2 \cdot 10^{29} \text {. }
$$

In the third application with $M=3.3 \cdot 10^{29}$, we get that $q_{68}$ satisfies the conditions of Lemma 1 and so $k<314$, which contradicts our assumption that $k>320$. This completes the proof of Theorem 1.

Acknowledgements. The authors are grateful to the anonymous referees for the careful reading the manuscript and the suggestions to improve the quality of this paper. 


\section{REFERENCES}

1. A. Baker, H. Davenport, The equations $3 x^{2}-2=y^{2}$ and $8 x^{2}-7=z^{2}$, Q. J. Math., 20 (1969), 129-137.

2. J.J. Bravo, J.L. Herrera, Repdigits in generalized Pell sequences, Arch. Math. (Brno), 56 (2020), 249-262.

3. J.J. Bravo, J.L. Herrera, F. Luca, On a generalization of the Pell sequence, Math. Bohem., 146 (2021), №2, 199-213.

4. J.J. Bravo, F. Luca, On the Diophantine equation $F_{n}+F_{m}=2^{a}$, Quaest. Math., 39 (2016), 391-400.

5. J.J. Bravo, C.A. Gómez, F. Luca, Powers of two as sums of two k-Fibonacci numbers, Miskolc Math. Notes, 17 (2016), 85-100.

6. J.J. Bravo, F. Luca, Powers of two as sums of two Lucas numbers, J. Integer Seq., 17 (2014), Article 14.8.3.

7. J.J. Bravo, F. Luca, Powers of two in generalized Fibonacci sequences, Rev. Colombiana Mat., 46 (2012), $67-79$.

8. Y. Bugeaud, F. Luca, M. Mignotte, S. Siksek, Fibonacci numbers at most one away from a perfect power, Elem. Math., 63 (2008), 65-75.

9. Y. Bugeaud, M. Mignotte, S. Siksek, Classical and modular approaches to exponential Diophantine equations I. Fibonacci and Lucas perfect powers, Ann. of Math., 163 (2006), 969-1018.

10. A. Dujella, A. Pethö, A generalization of a theorem of Baker and Davenport, Q. J. Math., 49 (1998), 291-306.

11. A. Gueye, S. Rihane, A. Togbé, Coincidence between k-Fibonacci numbers and products of two Fermat numbers, Bull. Braz. Math. Soc. (N.S.), (2021), doi: https://doi.org/10.1007/s00574-021-00269-2

12. B. Kafle, S. Rihane, A Togbé, A note on Mersenne Padovan and Perrin numbers, The Notes on Number Theory and Discrete Mathematics, 27 (2021), 161-170.

13. A.Ya. Khinchin, Continued Fractions, Noordhoff, Groningen, 1963.

14. E. Kiliç, On the usual Fibonacci and generalized order-k Pell numbers, Ars Combin., 88 (2008), 33-45.

15. E. Kiliç, The Binet formula, sums and representations of generalized Fibonacci p-numbers, European J. Combin., 29 (2008), 701-711.

16. E. Kiliç, D. Taşci, The generalized Binet formula, representation and sums of the generalized order-k Pell numbers, Taiwanese J. Math., 10 (2006), 1661-1670.

17. E.M. Matveev, An explicit lower bound for a homogeneous rational linear form in the logarithms of algebraic numbers, II, Izv. Math., 64(6) (2000), 1217-1269.

18. S.G. Sanchez, F. Luca, Linear combinations of factorials and $S$-units in a binary recurrence sequence, Ann. Math. Québec, 38 (2014), 169-188.

19. B.M.M. de Weger, Algorithms for Diophantine equations, PhD Thesis, Eindhoven University of Technology, Eindhoven, the Netherlands, 1989.

Department of Mathematics, University of Ghana

Department of Mathematics and Computer Sciences

University Center Abdelhafid Boussouf

Mila, Algeria

Department of Mathematics and Statistics

Purdue University Northwest

Westville, USA

bvnormenyo@ug.edu.gh, salahrihane@hotmail.fr, atogbe@pnw.edu 